\title{
Análise da ceratometria corneana em pacientes portadores de espasmo hemifacial
}

\section{Analysis of corneal keratometry in patients with hemifacial spasm}

Ivana Cardoso Pereira', Patrícia Grativol Costa Saraiva², Fabio Petersen Saraiva ${ }^{3}$, Fabricio Witzel de Medeiros ${ }^{4}$, Gustavo Miki ${ }^{5}$, Suzana Matayoshi ${ }^{6}$

\section{RESUMO}

Objetivo: Determinar a variação da ceratometria em olhos de pacientes portadores de espasmo hemifacial submetidos a tratamento com toxina botulínica. Métodos: Foram incluídos 18 pacientes portadores de espasmo hemifacial que foram submetidos ao exame oftalmológico completo, além da topografia corneana e Orbscan previamente à aplicação de toxina botulínica tipo A e 1 mês após o procedimento. Resultados: Não houve diferença estatisticamente significante entre a ceratometria encontrada pela topografia corneana ou diferença no BFS ("best fit sphere") anterior e posterior e índice de Roush avaliados pelo Orbscan nos olhos acometidos pelo espasmo em relação ao olho contralateral quando comparados antes e após o procedimento. Conclusão: Neste estudo, não encontramos diferença entre olhos com espasmo hemifacial e olhos sem essa condição quando avaliados a ceratometria, o índice de Roush e o BFS corneano antes e após tratamento.

Descritores: Espasmo hemifacial/complicações; Ceratometria corneana; Toxina botulínica tipo A/uso terapêutico; Topografia da córnea/métodos

\footnotetext{
'Médica estagiária do Setor de Plástica Ocular do Departamento de Oftalmologia do Hospital das Clínicas da Universidade de São Paulo - (USP) - São Paulo (SP), Brasil;

²Pós-graduanda (Doutorado) do Departamento de Oftalmologia do Hospital das Clínicas da Universidade de São Paulo - (USP) - São Paulo (SP), Brasil;

${ }^{3}$ Pós-graduando (Doutorado) do Departamento de Oftalmologia do Hospital das Clínicas da Universidade de São Paulo - (USP) - São Paulo (SP), Brasil;

${ }^{4}$ 'Fellow' do Setor de Córnea e Cirurgia Refrativa - The Cole Eye Institute - The Cleveland Clinic Foundation - Cleveland - USA; ${ }^{5}$ Médico Residente de terceiro ano - Departamento de Oftalmologia do Hospital das Clínicas da Universidade de São Paulo (USP) São Paulo (SP), Brasil;

${ }^{6}$ Médica Assistente do setor de Plástica Ocular do Departamento de Oftalmologia do Hospital das Clínicas da Universidade de São Paulo - (USP) - São Paulo (SP), Brasil

Trabalho desenvolvido junto ao Departamento de Oftalmologia do Hospital das Clínicas da Universidade de São Paulo (USP) - São Paulo (SP), Brasil em conjunto com a Cleveland Clinic Foundation (The Cole Eye Institute - USA)
}

Os autores declaram inexistir conflitos de interesse

Recebido para publicação em 5/11/2011 - Aceito para publicação em 18/4/2011 


\begin{abstract}
Purpose: To determine the corneal keratometric variation in patients' eyes with unilateral hemifacial spasm which underwent treatment with botulinum toxin and set against control group. Methods: Eighteen patients with hemifacial spasm were submitted to complete ophthalmologic exam, corneal topography and Orbscan previously botulinum toxin application and one month afterwards. They were evaluated for keratometry, BFS (anterior and posterior) and Roush value. Results: There were no statistical differences between eyes with hemi facial spasm and normal eyes related to keratometric values by topography and in the parameters evaluated by Orbscan as Roush and best fit sphere (anterior or posterior) prior to or after treatment with botulinum toxin. Conclusion: This study has not shown statistical difference between the corneal curvatures when compared eyes with hemi facial spasm with the opposite normal eyes.

Keywords: Hemifacial spasm/complications; Corneal keratometry; Botulinum toxin, type A/therapeutic use; Corneal topography/methods
\end{abstract}

\section{INTRODUÇÃO}

A s discinesias craniofaciais que são um grupo de doenças caracterizadas por movimentos involuntários da face, língua, palato, faringe, cordas vocais e pescoço classificam-se em: blefaroespasmo essencial, espasmo hemifacial, Síndrome de Meige, Síndrome de Brueghel, entre outras ${ }^{(1)}$.

Os espasmos hemifaciais são contrações tônicas ou clônicas involuntárias, paroxísticas, limitadas aos músculos de um lado do rosto, inervados pelo nervo facial. A ressonância magnética pode mostrar compressão vascular da raiz do $7^{\circ}$ nervo ${ }^{(2)}$. Inicia-se geralmente com tremores palpebrais intermitentes em uma pálpebra e, em meses ou anos, há progressão que envolve áreas adjacentes inervadas pelo nervo facial, comprometendo todos os músculos do lado acometido da face ${ }^{(3)}$.

A verdadeira causa do astigmatismo corneano ainda é incerta. Desde que Snellen, no século XIX, propôs que a pálpebra pode estar relacionada com a ocorrência do astigmatismo, muitos estudos foram iniciados ${ }^{(4)}$.

Existem várias evidências de que a compressão das pálpebras influencia a biomecânica corneana. O calázio, por exemplo, em pálpebra superior produz um achatamento corneano reversível ${ }^{(5)}$; o mesmo ocorre em pacientes portadores de peso de ouro em pálpebra superior ${ }^{(6)}$.

Neste estudo, investigamos a ocorrência de alterações nos valores ceratométricos, "best fit sphere" (anterior e posterior) e índice de Roush em pacientes portadores de espasmo hemifacial, comparando a curvatura corneana do olho acometido com a do olho contralateral e a influência do tratamento com toxina botulínica.

\section{Métodos}

Foi realizado um estudo prospectivo onde 18 pacientes portadores de espasmo hemifacial foram triados no Ambulatório de Plástica Ocular do HCFMUSP.Os pacien- tes foram submetidos à aplicação de toxina botulínica tipo $\mathrm{A}\left(\mathrm{Botox}^{\circledR}\right)$.Esta toxina é um preparado liofilizado, em que cada frasco contém 100U de droga, devendo permanecer no congelador, à temperatura de $-5^{\circ} \mathrm{C}$ até sua utilização. A droga foi reconstituída em $4,0 \mathrm{ml}$ de soro fisiológico, resultando numa concentração de $2,5 \mathrm{U}$ da toxina em cada $0,1 \mathrm{ml}$ da solução. Em cada ponto foram injetadas 2,5U nos músculos protractores das pálpebras (músculos orbicular, próceros e corrugadores de supercílios) e em outros músculos faciais (zigomático e orbicular da boca).

Todos os pacientes já vinham recebendo este tipo de tratamento com intervalo de aproximadamente 6 meses entre as aplicações. Foram excluídos do estudo os pacientes que já haviam sido submetidos à cirurgia ocular prévia, além dos pacientes que possuíam alterações topográficas compatíveis com ectasias corneanas.

Foram analisados os seguintes dados: idade; sexo; tempo de doença; uso de medicação sistêmica; acuidade visual sem correção; refração dinâmica; exame de topografia corneana e topografia de varredura de fenda de luz (Orbscan ${ }^{\mathrm{R}}$, Baush \& Lomb - Orbtek Inc, Salt Lake City, Utah). Os exames foram realizados momentos antes da aplicação da toxina botulínica e repetidos após um mês.

O exame de topografia foi realizado em todos os casos pelo mesmo examinador, utilizando a videoceratoscopia computadorizada modelo "Eye Sys" (Eye Sys Laboratories, Inc, Houston, Texas, EUA), levando em consideração a ceratometria média e o mapa de cores.

$\mathrm{O}$ exame de Orbscan também foi realizado em todos os casos pelo mesmo examinador, sendo comparados os parâmetros de melhor esfera adaptável nas superfícies anterior e posterior ("best fit sphere"), além da determinação do índice de Roush antes e após o tratamento, nos olhos tratados e não tratados.

Os resultados foram comparados utilizando-se o teste $t$ de Student para duas amostras em par para médias, com um nível de significância menor a 0,05 (5\%). 


\section{Gráfico 1}

Distribuição do índice de Roush (IR) entre os olhos dos 18 pacientes do estudo (eixo $\mathrm{X}$ )

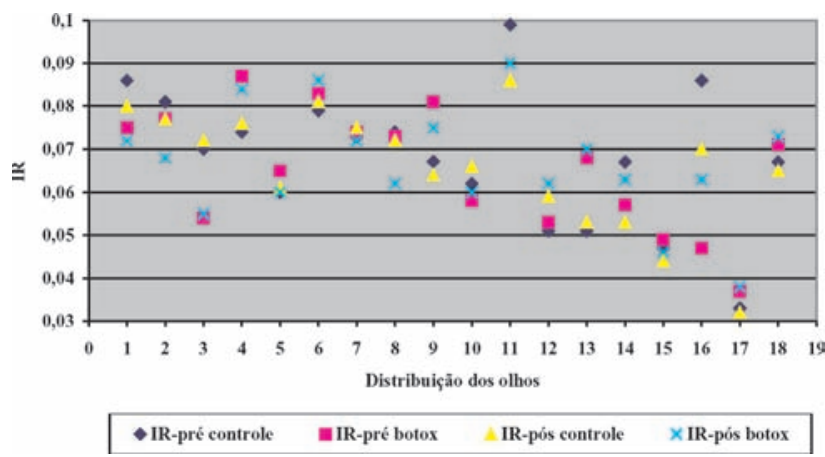

Gráfico 3

Distribuição dos valores relacionados ao "posterior best fit sphere" obtido com o Orbscan antes e após tratamento com toxina botulínica (tox). Não houve disparidade entre os grupos no pré e pós-procedimento $(p=0,30$ e $p=0,14$, respectivamente)

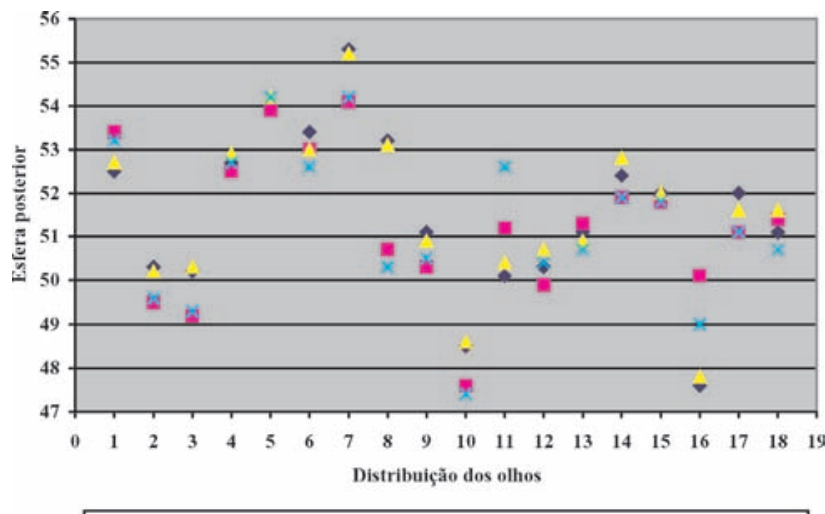

४ esf post pré controle Elesf post pré tox A esf post pós controle $X$ esf post pós tox

\section{Resultados}

O grupo era constituído de 10 pacientes do sexo feminino $(55,56 \%)$ e 08 pacientes do sexo masculino $(44,44 \%)$. A duração dos espasmos variou entre 1 a 25 anos, sendo que $50 \%$ dos pacientes apresentavam a doença há mais de 10 anos.

Foram comparadas as diferenças entre as medidas de ceratometria média central entre os dois olhos nos pacientes portadores de espasmo hemifacial, levando em consideração os valores obtidos nos exames de Topografia corneana.

\section{Gráfico 2}

Evolução da melhor esfera anterior adaptada ("best fit sphere") nos pacientes do estudo, $p=0,08$ prétoxina e $p=0,051$ pós-toxina botulínica quando comparados os olhos em estudo com seus controles

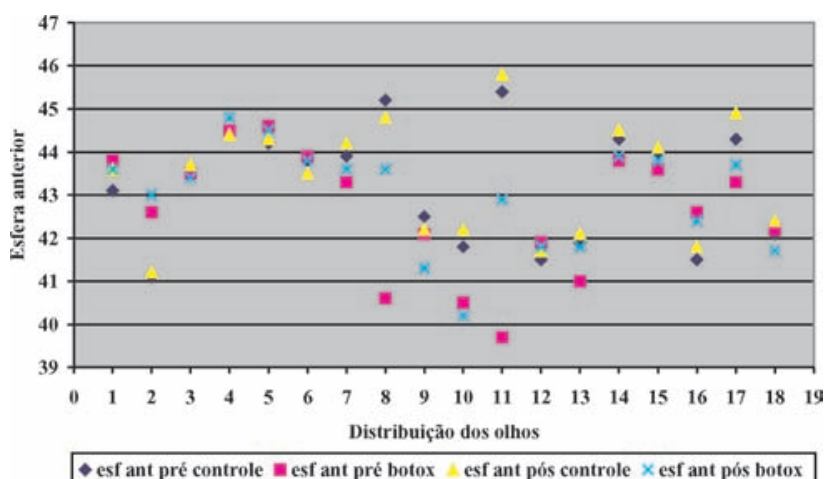

\section{Gráfico 4}

Ceratometria central $(K)$ medida pelo exame de topografia corneana pré e pós-toxina botulínica (tox). Não há diferença estatística entre os valores obtidos pré e pós-injeção de toxina botulínica $(p=0,44$ e $p=0,20$, respectivamente)

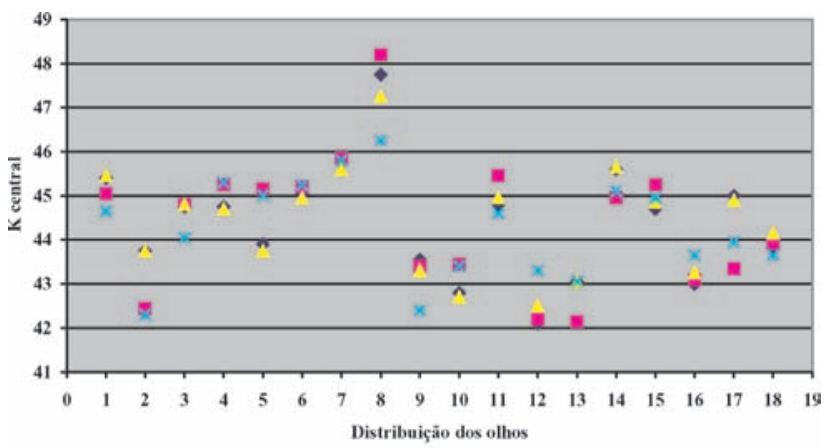

$\bullet K$ central pré controle $\mathbf{E}$ central pré tox $K$ Kentral pós controle $\times K$ central pós tox

Os olhos que foram submetidos ao tratamento com toxina botulínica não apresentaram diferença estatisticamente significante previamente ao uso da medicação quando comparados ao grupo controle em relação a ceratometria central, BFS anterior, BFS posterior e índice de Roush ( $\mathrm{p}=0,44 ; \mathrm{p}=0,08 ; \mathrm{p}=0,30$ e $\mathrm{p}=0,42$ respectivamente)

O gráfico 1 demonstra os resultados obtidos através do exame de Orbscan com os determinados índices de Roush pré e pós-procedimento para os pacientes arrolados no estudo. $\mathrm{O}$ índice de Roush não apresentou diferença entre os grupos após o procedimento terapêutico $(\mathrm{p}=0,17)$. 
A melhor esfera anterior adaptada ("anterior best fit sphere") por sua vez mostrou uma tendência de diminuição dos valores de curvatura corneana após a aplicação de toxina botulínica quando comparado com o controle embora não tenha sido significante da mesma forma que os outros parâmetros $(\mathrm{p}=0,051)$. Os dados estão representados no gráfico 2.

Melhor esfera posterior adaptada ("posterior best fit sphere") também não apresentou disparidade entre os grupos no pós-procedimento $(\mathrm{p}=0,14)$. $\mathrm{O}$ gráfico 3 mostra os resultados.

Não houve diferença estatisticamente significante após o procedimento entre os olhos acometidos pelo espasmo e o grupo controle em relação à ceratometria central avaliada pela topografia corneana $(\mathrm{p}=0,20)$. $\mathrm{O}$ gráfico 4 revela a evolução dos resultados em relação à ceratometria.

\section{Dıscussão}

As distonias faciais constituem um grupo de alterações neuromusculares, que acometem particularmente os músculos palpebrais e/ou músculos faciais. No espasmo hemifacial há contrações musculares involuntárias e periódicas em um dos lados da face, levando a modificação da expressão do paciente do lado afetado(2).

No grupo estudado houve uma maior prevalência de pacientes do sexo feminino e com uma idade média de 64 anos, concordando com outros autores que encontraram uma maior prevalência em mulheres e em uma faixa etária entre 60 e $80 \operatorname{anos}^{(2,6-8)}$.

O uso de toxina botulínica para o tratamento das distonias faciais está se ampliando nos últimos anos. A toxina botulínica é um tipo de neurotoxina elaborada pelo Clostridium botulinum. Existem diversos tipos de toxina botulínica na natureza e, dentre eles, o tipo A é o mais estável e usado comercialmente em medicamentos ${ }^{(9)}$. Ela age por bloqueio seletivo e reversível da junção neuromuscular, causando relaxamento da musculatura envolvida. A toxina inibe a liberação de acetilcolina das vesículas pré-sinápticas da placa neural, provocando uma neurectomia transitória ${ }^{(2,3)}$.

As causas do astigmatismo corneano não são totalmente conhecidas, porém vários fatores podem contribuir com o desenvolvimento corneano e a compressão exercida pelas pálpebras deve ser considerada como um dos mais importantes fatores ${ }^{(4)}$. Ugurbas e Zilelioglu demonstraram a ocorrência de um astigmatismo assimétrico em pacientes portadores de ptose congênita unilateral ${ }^{(10)}$, enquanto Lavy et al. demonstraram uma alteração no astigmatismo corneano em pacientes portadores de lagoftalmo e submetidos ao implante de peso de ouro ${ }^{(11)}$.

Estudo realizado por Moon et al. encontrou, em pacientes portadores de espasmo hemifacial, uma mai- or ocorrência de astigmatismo a favor da regra no lado acometido em relação ao lado contralateral ${ }^{(4)}$.

Nosso estudo não demonstrou uma diferença estatisticamente significativa entre a curvatura corneana presente no lado acometido em relação ao lado contralateral. Alguns fatores aqui podem ter influenciado os resultados como o fato de os pacientes já possuírem um prévio histórico de tratamento com toxina botulínica, influenciando as possíveis alterações geradas pelo fenômeno de compressão do espasmo. Um estudo prospectivo incluindo pacientes virgens de prévio tratamento com toxina poderia elucidar essa questão.

\section{Conclusões}

Não encontramos diferença estatisticamente significativa entre os parâmetros de ceratometria central, BSF anterior e posterior e índice de Roush avaliados pelos métodos de topografia corneana e Orbscan quando comparados olhos com espasmo hemifacial unilateral em relação ao olho contralateral.

\section{REFERÊNCIAS}

1. Cunha MC, Aguirre OP, Dias CRS. Tratamento do espasmo facial unilateral com toxina botulínica tipo A. Arq Bras Oftalmol. 1998;61(1):54-60.

2. Schellini SA, Matai O, Igami TZ, Padovani CR, Padovani CP. Blefaroespasmo essencial e espasmo hemifacial: características dos pacientes, tratamento com toxina botulínica A e revisão da literatura. Arq Bras Oftalmol 2006;69(1):23-6.

3. Carvalho RMLS, Gomi CF, Carvalho ALS, Matayoshi S, Moura EM. Tratamento do blefaroespasmo e distonias faciais correlatas com toxina botulínica: estudo de 16 casos. Arq Bras Oftalmol. 2003;66(1):13-6.

4. Moon NJ, Lee HI, Kim JC. The changes in corneal astigmatism after botulinum toxin-A injection in patients with blepharospasm. J Korean Med Sci. 2006;21(1):131-5.

5. Cosar CB, Rapuano CJ, Cohen EJ, Laibson PR. Chalazion as a cause of decreased vision after LASIK. Cornea. 2001;20(8):890-2.

6. Novak KD, Kohnen T, Chang-Godinich A, Soper BA, Kennedy $\mathrm{P}$, Wang Q, et al. Changes in computadorized videokeratography induced by artificial tears. J Cataract Refract Surg. 1997;23(7):1023-8.

7. Grandas F, Elston J, Quinn N, Marsden CD. Blepharospasm: a review of 264 patients. J Neurol Neurosurg Psychiatry. 1998:51(6):767-72.

8. Costa PG, Pereira IC, Saraiva FP, Raiza ACP, Tanaka LK, Matayoshi S. Avaliação do filme lacrimal de pacientes com distonia facial durante tratamento com toxina botulínica tipo A. Arq Bras Oftalmol. 2006;69(3):319-22.

9. Waller RR, Kennedy RH, Henderson JW, Kesty KR. Management of blepharospasm. Trans Am Ophthalmol Soc. 1985;83:367-86.

10. Uðurbap SH, Zilelioðlu G. Corneal topography in patients with congenital ptosis. Eye (Lond). 1999;13(Pt 4):550-4.Lavy JA, East CA, Bamber A, Andrews PJ. Gold weight implants in the management of lagophthalmos in facial palsy. Clin Otolaryngol Allied Sci. 2004;29(3):279-83. 